\title{
Energy-efficient User Association in Cognitive Heterogeneous Networks
}

\author{
Agapi Mesodiakaki, Ferran Adelantado, Luis Alonso, and Christos Verikoukis
}

\begin{abstract}
Due to the ever-increasing data traffic demands, which are directly connected to increased energy consumption, it becomes challenging for operators to achieve capacity enhancement, while limiting their electric bill. To that end, exploiting the context-awareness of future cognitive networks is expected to play a key role. Next generation cellular networks are about to include a plethora of small cells (SCs), with users being able of communicating via multiple bands. Given that SCs are expected to be eventually as close as $50 \mathrm{~m}$ apart, not all of them will have a direct connection to the core network, and thus, multi-hop communication through neighboring SCs may be required. In such architectures, the user association problem becomes challenging, with backhaul (BH) energy consumption being a definitive parameter. Thus, in this article, we study the user association problem in cognitive heterogeneous networks. We evaluate the existing approaches in terms of energy efficiency and we show the potential of exploiting the available context-aware information (i.e., users' measurements and requirements, the knowledge of the network architecture and the available spectrum resources of each base station) to associate the users in an energy-efficient way, while maintaining high spectrum efficiency. Our study considers both the access network and BH energy consumption, while the performance of the association algorithms is evaluated under two different case study scenarios.
\end{abstract}

\section{INTRODUCTION}

Nowadays, due to the increased need for anywhere-anytime connectivity, the mobile data traffic demands are growing exponentially. Thus, it becomes urgent for mobile operators to achieve sustainable capacity improvement. In Long Term Evolution (LTE)/LTE-Advanced (LTE-A) networks, the achievable capacity is highly dependent on the channel quality. Due to the use of link adaptation, the modulation and coding scheme (MCS) is adapted to the signal to interference plus noise ratio (SINR). Therefore, for higher SINR, a MCS of higher order is used, resulting in higher achievable capacity. However, what still remains a challenge is how to guarantee a high SINR. A straightforward solution is to bring the transmitter closer to the receiver, while at the same time mitigating the received interference. Hence, a significant densification of the cellular networks deployment is expected the forthcoming years.

The benefit of adding more base stations (BSs) is twofold: i) the distance between user equipments (UEs) and BSs is reduced and thus the SINR increases and ii) each UE shares the BS's bandwidth and backhaul $(\mathrm{BH})$ connection with a smaller number of UEs, thus gaining access to a larger portion of resources, which results in extra capacity improvement. However, adding more macrocell BSs (i.e., eNodeBs (eNBs) in the LTE terminology) to the network is not viable in many important markets due to the cost and the lack of available sites and proper connections among them. Consequently, future cellular networks are expected to be dense deployments of BSs of widely varying transmit powers and coverage areas (i.e., eNBs and/or small cells (SCs)), carrier frequencies, BH connection types and communication protocols. Besides their heterogeneous nature, they will be also characterized by the implementation of cognitive capabilities in some of the involved network entities. In particular, they will be able to perceive current network conditions (i.e., context-awareness), plan, decide and act according to these conditions, the so-called self-organized networks (SONs), learn from the consequences of their actions, while aiming

A. Mesodiakaki and L. Alonso are with the Signal Theory and Communications Department, Technical University of Catalonia, Spain. E-mail:\{agapi.mesodiakaki, luisg\}@tsc.upc.edu

F. Adelantado is with Open University of Catalonia, Barcelona, Spain. E-mail: ferranadelantado@uoc.edu

C. Verikoukis is with Telecommunications Technological Centre of Catalonia, Castelldefels, Spain. E-mail: cveri@cttc.es 
at achieving specific goals. Therefore, in the rest of the article, we will refer to these networks as cognitive heterogeneous networks (HetNets).

In next generation cognitive HetNets, UEs will be capable of communicating via multiple bands over various protocols and thus the user association problem becomes challenging, with future HetNet architecture playing an important role. As SCs are expected to be eventually as close as $50 \mathrm{~m}$ apart [1], it is expected that not all of them will have a direct connection to the core network (CN). To that end, LTE-A [2] considers that a SC may be connected to the $\mathrm{CN}$ through a gateway, known as Home eNB gateway (HeNB GW). NGMN Alliance [3] and Tellabs [4] also consider an optional aggregation gateway to be introduced for better scalability (e.g., reducing the number of logical S1 interfaces to be supported by the $\mathrm{CN}$ ). Moreover, assuming that the operator already has a radio access network in place, a straightforward option is to connect the SC directly to the eNB site, especially in cases where there is fiber access available [3], [4]. From topology perspective, this would look like a traditional hub-and-spoke, with the eNB as hub and the SCs as spokes, while ring, chain or mesh topologies can be used between SCs to provide further connectivity, as depicted in Fig. 1 [4].

In such topologies, where one SC backhauls its traffic to the neighboring SC that acts as an aggregation point, the extension and configuration of the $\mathrm{BH}$ involves a multidimensional trade-off between the reduction of the capital expenditure (CAPEX), the reduction of the energy consumption, with the consequent reduction of operational expenditure (OPEX), and the maximization of the access network capacity. It is precisely in this context that, for a BH configuration, which is often determined by external constraints, the user association strategy should exploit context-awareness to deal with the aforementioned complexity.

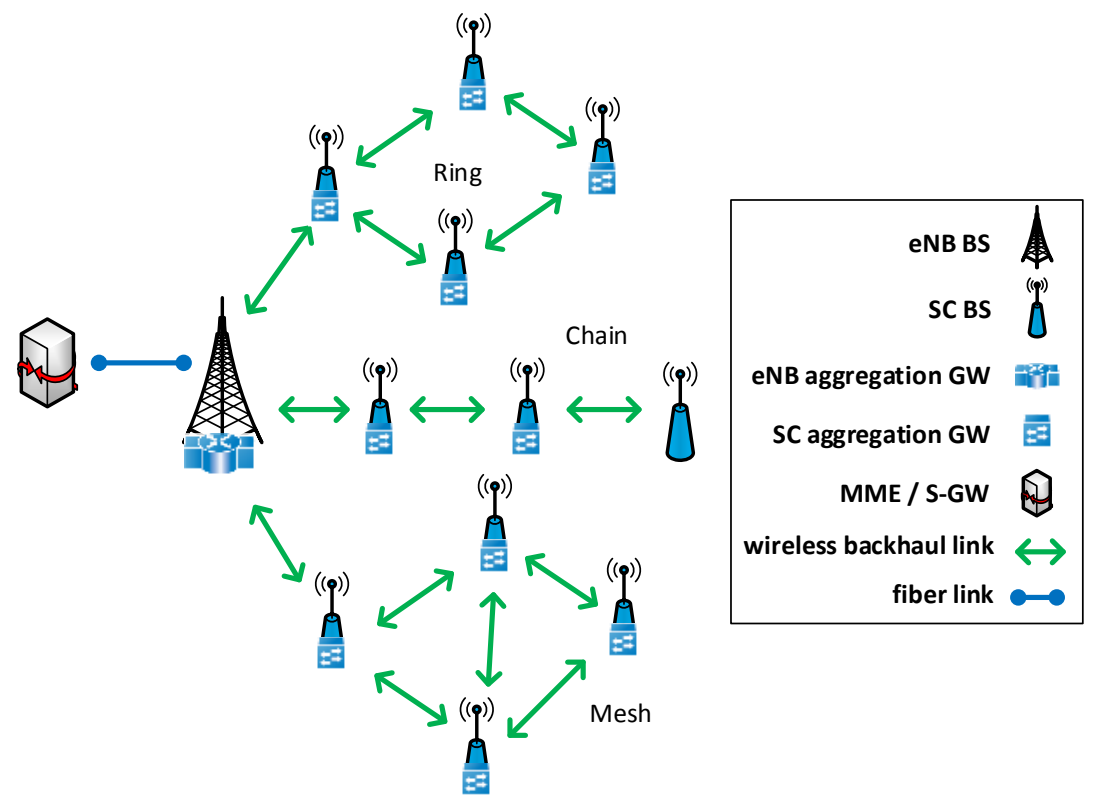

Fig. 1. Future cognitive HetNet topology.

Thus, in this article, we focus on next generation cognitive HetNets and we study the user association problem in scenarios where several SCs backhaul their traffic to the neighboring cells until they reach the $\mathrm{CN}$. We evaluate the performance of the existing user association algorithms and we show the high energy efficiency potential of a cognitive algorithm that exploits the available context-aware information (i.e., UE measurements and requirements, the HetNet architecture knowledge and the available spectrum resources of each BS) to associate the UEs in an energy-efficient way.

The rest of the article is organized as follows: In Section II, the main technical challenges in future cognitive HetNet user association are studied and analyzed. In Section III, the current user association algorithms proposed in the literature along with a new context-aware user association algorithm are discussed. Section IV includes the performance comparison of the user association algorithms, where 
useful insights and conclusions are provided. Finally, Section VII concludes the article.

\section{TECHNICAL CHALLENGES}

The main objective of user association in future cognitive HetNets is to achieve network capacity enhancement, while satisfying the UE QoS requirements. To that end, three main challenges ${ }^{1}$ can be identified: interference management, spectrum efficiency maximization and energy efficiency maximization.

\section{A. Interference Management}

As future HetNets become denser and spectrum resources are limited, high spectrum reuse is necessary to achieve higher spectrum efficiency. Under spectrum reuse, interference management becomes a major concern. User association in future HetNets imposes additional challenges on the interference management, since a UE may communicate with a different BS than the one from which it receives the strongest signal. Thus, in this case, the UE experiences strong downlink interference, while causing high uplink interference to UEs in its proximity. Furthermore, the complex $\mathrm{BH}$ architecture should be taken into account to efficiently avoid/mitigate the generated interference.

Several interference management techniques have been proposed so far. Although fractional frequency reuse has been traditionally used, it presents low efficiency, while its complexity increases when applied in dense HetNets consisting of many tiers. Thus, in releases 8/9 of LTE, inter-cell interference coordination (ICIC) was introduced, where the inter-cell interference is controlled by radio resource management (RRM) methods based on the cell spectrum usage and traffic load [2]. In the enhanced ICIC (eICIC), introduced in release 10 of LTE-A, the transmissions of multiple cells are coordinated not only in the frequency domain but also in the time domain using power control schemes. Furthermore, aiming at improving the interference management, eICIC introduced the almost blank subframes (ABS), during which the eNB remains silent (i.e., only transmitting control information at very low power), thus enabling the UE communication with their associated SCs without interference caused by the eNB [2].

In general, in future cognitive HetNets the user association and the interference management constitute two interdependent problems and therefore, should be jointly studied and designed.

\section{B. Spectrum Efficiency Maximization}

Due to the previously explained spectrum scarcity problem, the spectrum efficiency maximization (i.e., maximization of the achievable data for a given spectrum band) is another challenge mobile operators have to meet. This metric is directly connected to the achieved SINR. Specifically in LTE-A networks, the spectrum efficiency of a UE is a scalar step function of its SINR, with each step corresponding to the use of a specific MCS and thus to a specific achievable rate [5].

Network densification is expected to improve the overall spectrum efficiency, since the distance between UEs and BSs decreases, and thus higher SINR is achieved. In LTE, further spectrum efficiency enhancement is provided by Multiple-Input Multiple Output (MIMO) technology and carrier aggregation. MIMO, which uses multiple antennas at both the transmitter and receiver, offers significant increases in data throughput and link range without additional bandwidth or increased transmit power. On the other hand, through carrier aggregation higher data rates are achieved, since the overall used bandwidth increases.

In future cognitive HetNets, a UE may not be always connected to the BS from which it receives the highest SINR. Hence, spectrum efficiency becomes even more challenging, stressing the need for spectrum-aware user association strategies.

\footnotetext{
${ }^{1}$ Resource allocation fairness is another main HetNet challenge. Yet, it has not been included, since it is a cross-cutting objective that encompasses the three main challenges discussed in this article and all the radio resource management functions, particularly scheduling.
} 


\section{Energy Efficiency Maximization}

Maximizing the network energy efficiency may be defined as maximizing the successfully sent data while minimizing the total energy consumption. In case of specific UE requirements, this can be expressed as satisfying the UE traffic demands, while minimizing the total energy consumption.

The total energy consumption is the sum of the energy consumed in the access network, i.e., between the UE and the BS (Uu interface) and in the BH links i.e., between BSs and/or the $\mathrm{CN}$. The BH energy consumption definitely impacts the overall energy efficiency, especially when considering scenarios as the ones previously described with BH links, and thus cannot be neglected.

Given the common assumption that the total transmit power of a BS is equally distributed among its subcarriers [6], the energy consumed in the access network is a function of the number of resource block pairs (RBPs) needed to serve the UE traffic. The more the RBPs allocated to the UE, the higher the access network energy consumption to serve the UE traffic. Also note that as eNBs have a much higher total transmit power than SCs, the power allocated to an RBP, and thus the access network energy consumption, is higher when a UE is associated with an eNB than with a SC.

Then, the BH link energy consumption is a scalar function of the aggregated throughput that passes through the link (i.e., the sum of the total throughput of all UEs associated with the SCs that backhaul their traffic through this link). Specifically, given that adaptive MCS is used, the energy consumption in a $\mathrm{BH}$ link can be expressed as a function of the aggregated throughput and thus of the required SINR to achieve this throughput. This function depends on the employed BH technology and the distance between the transmitter and the receiver of the BH link. Depending on the UE association, the BH energy consumption may vary significantly. The closer the serving BS to the $\mathrm{CN}$ (i.e., the less the number of hops until the data packets reach the $\mathrm{CN}$ ), the less the $\mathrm{BH}$ energy consumption. In addition, further energy efficiency gains can be achieved when BH load balancing is performed, since the energy consumption of a BH link does not increase linearly with its traffic load [5].

Overall, the network energy efficiency is highly dependent on the UE association decision, with $\mathrm{BH}$ energy consumption having a significant impact. As a result, user association algorithms taking into account both the access network and BH energy consumption should be designed.

\section{USER ASSOCIATION ALGORITHMS}

During the last few years, the UE association problem has received a lot of research attention. To that end, the most important user association algorithms may be summarized in the following ones:

\section{A. Reference signal received power (RSRP)}

In LTE-A, the user association is based on the reference signal received power (RSRP) and/or reference signal received quality (RSRQ). The first measures the average received power over the resource elements that carry cell-specific reference signals within certain frequency bandwidth, while the latter measures the portion of pure reference signal power over the total power received by the UE. Although these criteria maximize the SINR of UEs [7], simulations and field trials have shown that they do not increase the overall throughput as much as hoped, because many SCs typically have few active UEs. This stems from the fact that the transmit power of a SC is much lower with respect to the eNB, resulting to a smaller downlink SC coverage. However, this is not the case for the uplink, where the UE transmission range is the same for all UEs. The algorithm presents high spectrum efficiency, as the UE gets associated with the BS from which it receives the strongest signal. However, its energy efficiency cannot be guaranteed, since it only considers the radio access network.

\section{B. Range expansion (RE)}

RSRP presents relatively poor overall throughput performance, since most UEs get connected to the eNB, whereas many SCs have few active UEs. To that end, range expansion (RE) was proposed, which 
adds a bias (in $\mathrm{dB}$ ) to the RSRP in the case the signal comes from a SC, thus actively pushing UEs onto SCs [8], [9]. Despite a potentially significant SINR hit for that UE, this has the potential for a win-win situation, because the UE gains access to a much larger portion of resources, while the eNB reclaims the ones that would have been allocated to it. RE presents lower spectrum efficiency than RSRP, especially in cases an aggressive bias is used, as a UE is connected to a BS that does not provide the highest SINR. Finally, equivalently to RSRP, its energy efficiency cannot be guaranteed.

\section{Minimum path loss (MPL)}

A UE is connected to the BS from which it has minimum path loss (MPL), independently of its received power [6]. In ideal environments with no fading, the algorithm would connect the UE to its closest BS. In hotspot traffic distribution scenarios, MPL achieves maximum traffic offloading to SCs, since most UEs will be associated with SCs being in their proximity. Moreover, it achieves much lower spectrum efficiency than the maximum, as SINR is not taken into account in the user association decision. Although MPL minimizes the UE power consumption in uplink, it fails to guarantee high network energy efficiency, due to high $\mathrm{BH}$ energy consumption.

\section{Context-aware algorithm (CA)}

All the aforementioned approaches consider only the access network, thus totally overlooking any $\mathrm{BH}$ issues. Still, the $\mathrm{BH}$ is forecast to become the most challenging part for future SCs, especially when considering scenarios as the ones previously described with wireless BH links. In such scenarios, the $\mathrm{BH}$ energy consumption can be responsible for a high percentage of the total energy consumption and consequently cannot be ignored.

Thus, we propose a context-aware algorithm (CA), summarized in Fig. 2, that takes as input the available context-aware information (i.e., the UEs' measurements and requirements, the HetNet architecture knowledge and the available spectrum resources of each BS) to associate the UEs aiming at maximizing the network energy efficiency. Note that this context-aware information can be readily available to all nodes in a LTE-A network, while introducing low overhead (e.g., by transmitting it during the almost blank subframes (ABS) or through the X2 interface between BSs (eNBs and/or SCs) [2]).

At the same time, CA achieves high spectrum efficiency, since only the BSs that require the minimum number of RBPs to satisfy the UE requirements (i.e., the BSs with the best received SINR) are included in the UE candidate cell set. To ensure that all the UEs will be associated, CA sorts the UEs by their number of candidates and starts with the ones with the fewest candidates. Then, to maximize the network energy efficiency, CA sorts the candidate cells by the number of hops until their traffic reaches the CN and it associates the UE to the candidate with the fewest hops, as long as it has sufficient RBPs to serve it. Otherwise, it moves to the next candidate. Every time a UE is associated with a BS, the algorithm updates the remaining RBPs of the BS, its cell throughput and the throughput that passes through the $\mathrm{BH}$ links that are used until its traffic reaches the $\mathrm{CN}$. In the case there are more candidates with the same number of hops, CA associates the UE to the one with the least loaded BH route, as long as it has sufficient RBPs, to achieve load balancing at the BH links.

In terms of scalability, CA may be executed in each eNB sector at a specific time interval based on the dynamics of the UE traffic distribution, so that the system performance is optimized. For the new UEs that appear in the meantime, CA is executed as before given the associations of the rest of the UEs. However, in that case, the context-aware information includes the remaining RBPs and the throughput of each BS/cell, and the throughput that passes through each $\mathrm{BH}$ link, given the traffic of the already associated UEs.

\section{Performance Evaluation}

In this section, we evaluate the performance of the previously discussed user association algorithms in terms of spectrum efficiency and energy efficiency, aiming at gaining useful insights on their ability to meet future cognitive HetNet challenges. 


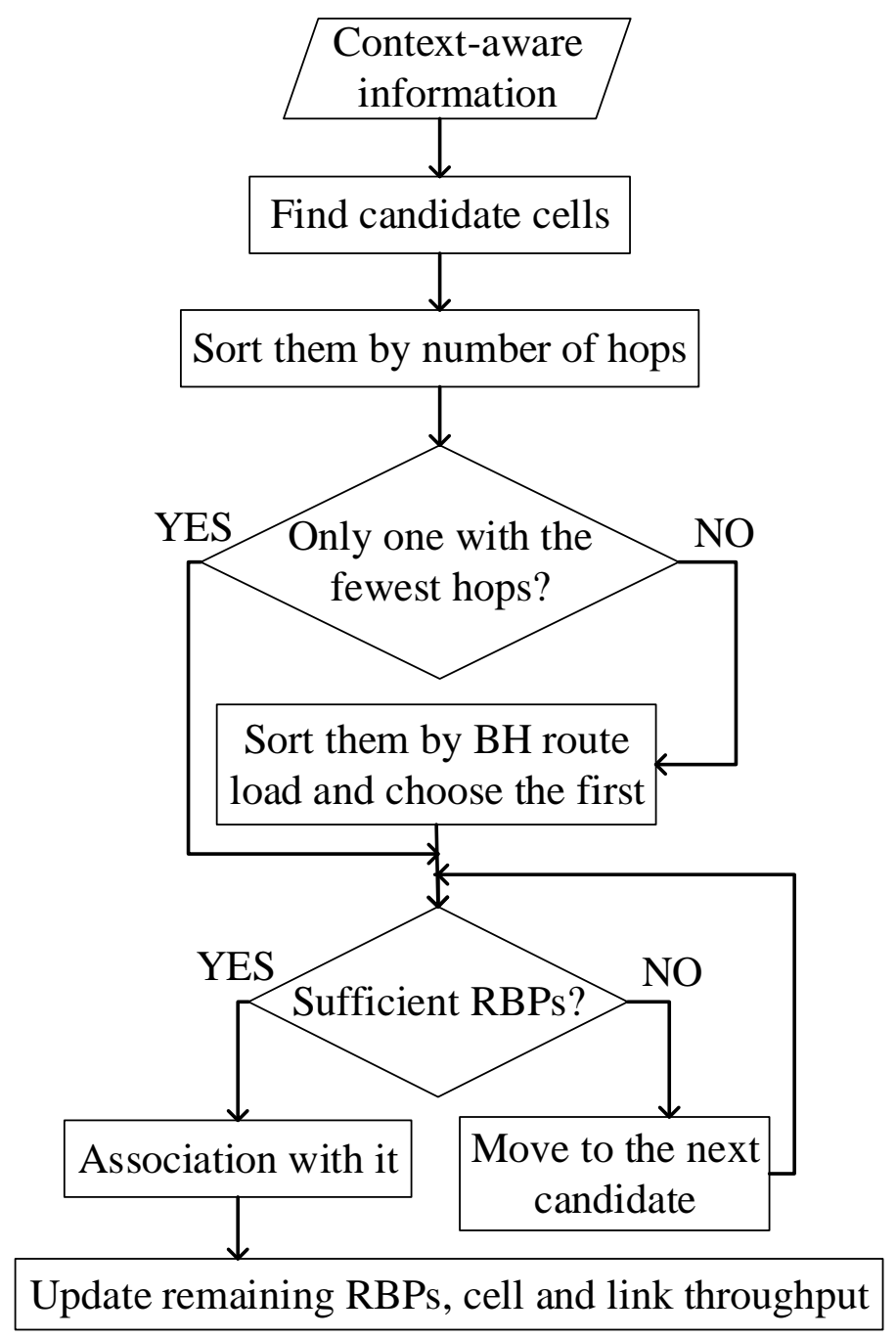

Fig. 2. Context-aware algorithm (CA) flowchart.

\section{A. Scenarios}

In our experiments, we consider a sector area of an eNB, which overlaps with 5 outdoor SCs. In order to study the impact of the $\mathrm{BH}$ architecture on the algorithm performance, two different $\mathrm{BH}$ architecture scenarios are employed, as depicted in Fig. 3(a) and 3(b). Notice that although the same BS deployment is considered in both scenarios, the $\mathrm{BH}$ architecture varies significantly (i.e., SCs 3 and 5 are connected through SC 4 in the second scenario). In general, this may happen due to deployment impairments (e.g., buildings), that impose restrictions on the actual $\mathrm{BH}$ link configurations.

The BH network consists of line-of-sight (LOS) millimeter wave (mmW) links (60 GHz band) with 50 $\mathrm{MHz}$ channel bandwidth [10], whereas the access network operates at $2.0 \mathrm{GHz}$ with $10 \mathrm{MHz}$ bandwidth being available to the eNB and to each SC. The association of the received SINR to the achievable spectral efficiency in bps/Hz can be found in [5, Table A.2], while the $\mathrm{mmW}$ link budget equation is given by [10, eq. 1.4]. In each simulation realization we considered $N$ fixed outdoor UEs of different throughput requirements. Specifically, $80 \%$ of UEs demand $500 \mathrm{kbps}, 10 \% 700 \mathrm{kbps}$ and $10 \% 1 \mathrm{Mbps}$. The maximum transmit power was equal to $46 \mathrm{dBm}$ for the eNB and $30 \mathrm{dBm}$ for a $\mathrm{SC}$, while path loss models and other setup parameters were selected according to 3GPP recommendations [11].

We further considered two user distribution scenarios. In the first (scenario 1), the UEs are uniformly distributed in the sector area of radius $R=450 \mathrm{~m}$, while in the second (scenario 2) they form hotspots. In particular, in scenario 2, we consider that $40 \%$ of the UEs are uniformly distributed in a radius $r=60$ $\mathrm{m}$ from SC 3, other $40 \%$ in a radius $r=60 \mathrm{~m}$ from SC 4 and the rest uniformly distributed in the whole 


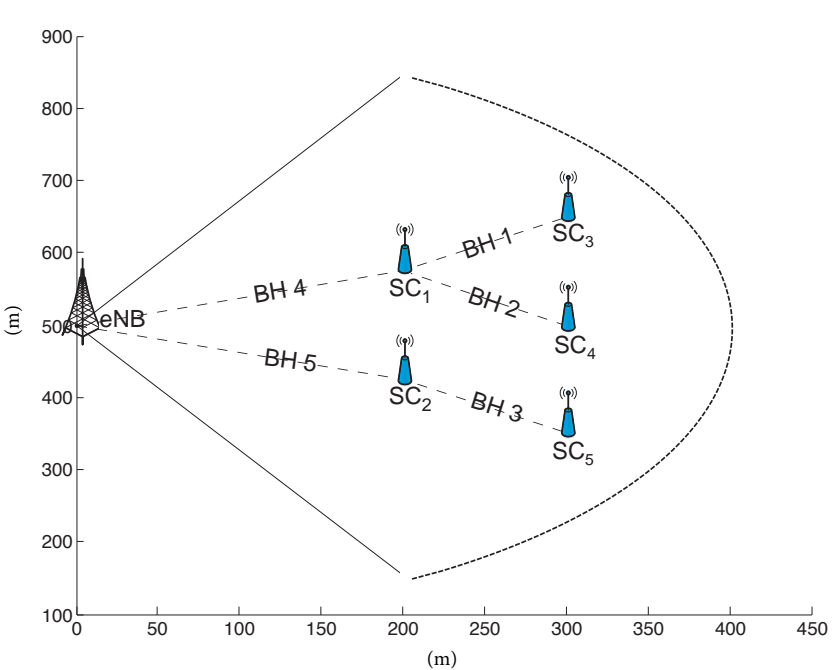

(a) $\mathrm{BH}$ architecture 1 .

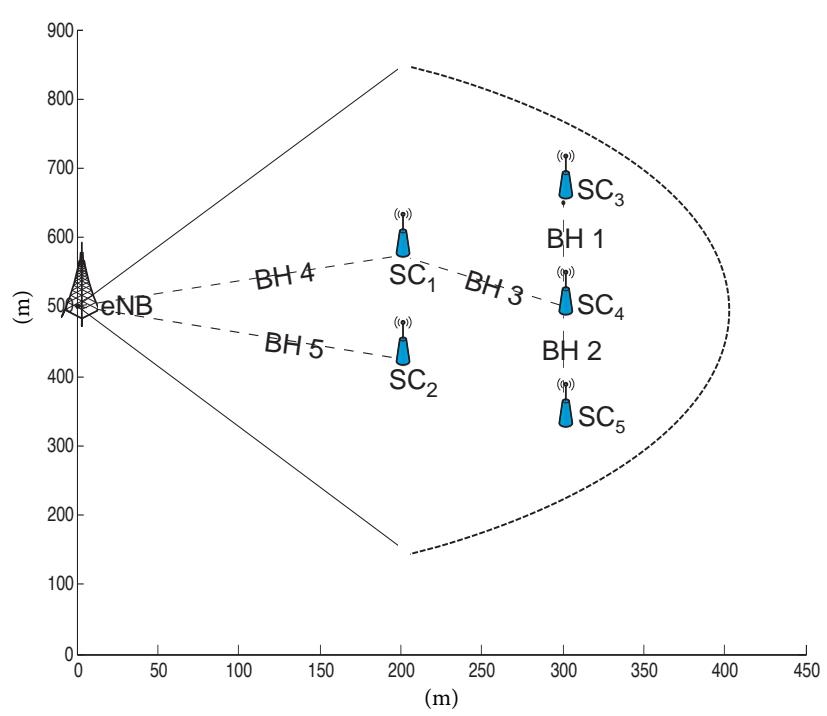

(b) $\mathrm{BH}$ architecture 2 .

Fig. 3. Simulation topology scenarios considering different $\mathrm{BH}$ architectures.

sector area. Note that this scenario constitutes a more realistic scenario, as in future HetNets the UEs are expected to be very close to SCs and to generate hotspot traffic of highly bursty nature [1]. The four user association algorithms, previously described in Section III, have been simulated in both scenarios.

\section{B. Results}

Maximizing the network energy efficiency becomes more challenging, as the number of UEs increases. Therefore, in our experiments, we consider highly loaded scenarios consisting of more than 60 UEs.

In terms of throughput, all algorithms achieve the same performance in both scenarios (e.g., for $N=100$ UEs, the total network throughput is $57 \mathrm{Mbps}$ ), as all algorithms ensure that UEs' demands are satisfied.

As far as the network energy efficiency of scenario 1 is concerned, CA achieves slightly better performance than RSRP for values higher than $N=70 \mathrm{UEs}$, as depicted in Fig. 4(a). This is due to the fact that CA gives priority to the candidate cell with the fewest hops and thus most UEs get connected to the eNB. In this case, the additional energy consumption experienced by $\mathrm{CA}$ in the Uu interface with respect to RSRP, is not compensated by the reduction of the BH energy consumption. However, for higher values, there are no available RBPs in the eNB at some point, and thus UEs are associated with the candidate SC with the fewest hops, which results in lower BH energy consumption compared to RSRP. This can be also noticed in Fig. 4(b), where the average traffic of each BH link of Fig. 3(a) is depicted, for $N=100$ UEs. According to it, $\mathrm{CA}$ achieves better load balancing among the $\mathrm{BH}$ links that are the same number of hops away from the $\mathrm{CN}$ (e.g., among $\mathrm{BH}$ links 4 and 5) than the other algorithms. Then, regarding RE, it achieves lower energy efficiency, since there are more UEs associated with SCs, resulting in higher BH energy consumption. Finally, MPL can be considered as an aggressive RE algorithm, where it is likely for the UEs to be closer to a SC and thus to be associated to it. Although MPL achieves maximum traffic offloading to SCs, it has very poor energy efficiency performance, as the BH traffic (see Fig. 4(b)) and thus the $\mathrm{BH}$ energy consumption is much higher than the other approaches.

In Fig. 4(c), the average network energy efficiency of all algorithms is depicted, when scenario 2 is applied. In this case, CA achieves gains up to $100 \%$, since it manages the BH traffic so that lower energy consumption is generated. Specifically, in comparison to RSRP, CA achieves up to $40 \%$ energy efficiency gain. This stems from the fact that the UEs located around SC 3 or 4, that have as candidate cells the SCs 1 or 2, will be associated with SC 3 or 4 when RSRP is applied, given that they receive higher SINR, whereas to SC 1 or 2 with CA, given that less hops are required to reach the CN. As a result, $\mathrm{CA}$ generates globally less traffic for the $\mathrm{BH}$ links and thus the $\mathrm{BH}$ energy consumption becomes much 


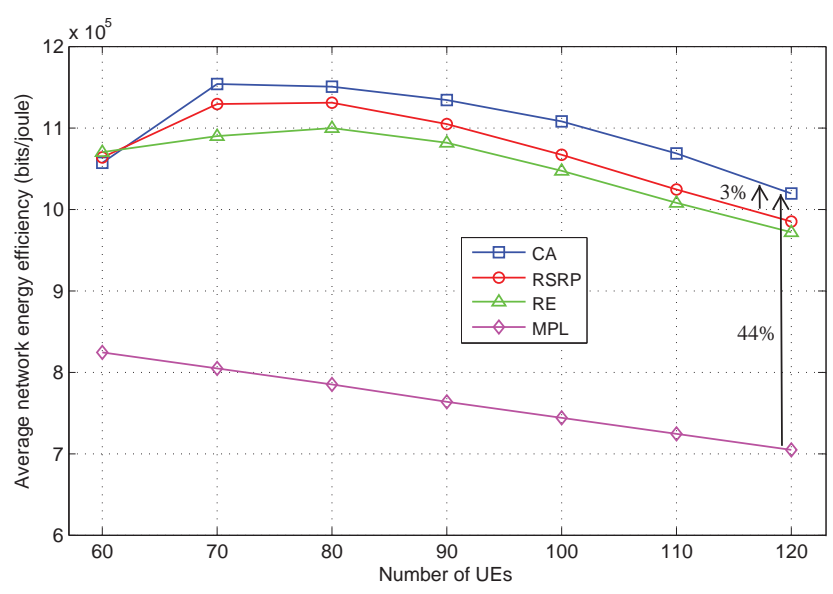

(a) Average network energy efficiency for different number of users $(N)$, when the UEs are uniformly distributed in the sector area (scenario 1).

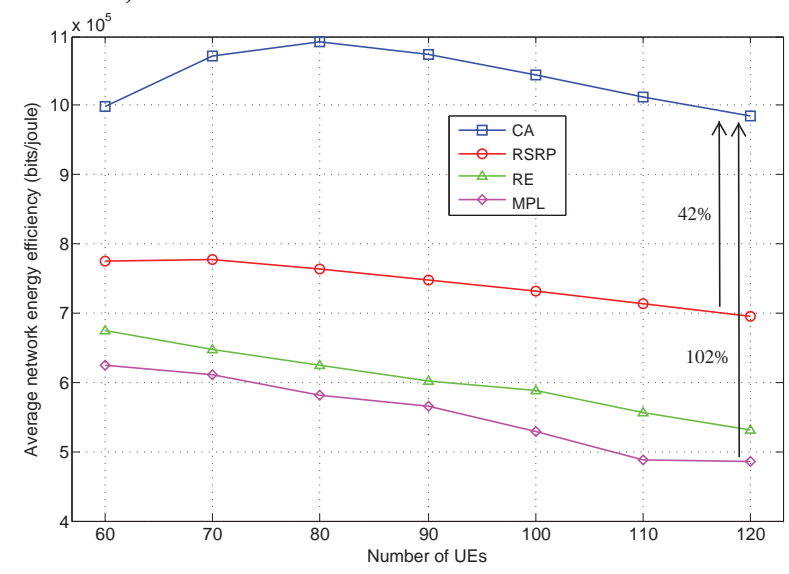

(c) Average network energy efficiency for different number of users $(N)$, for hotspot traffic (scenario 2).

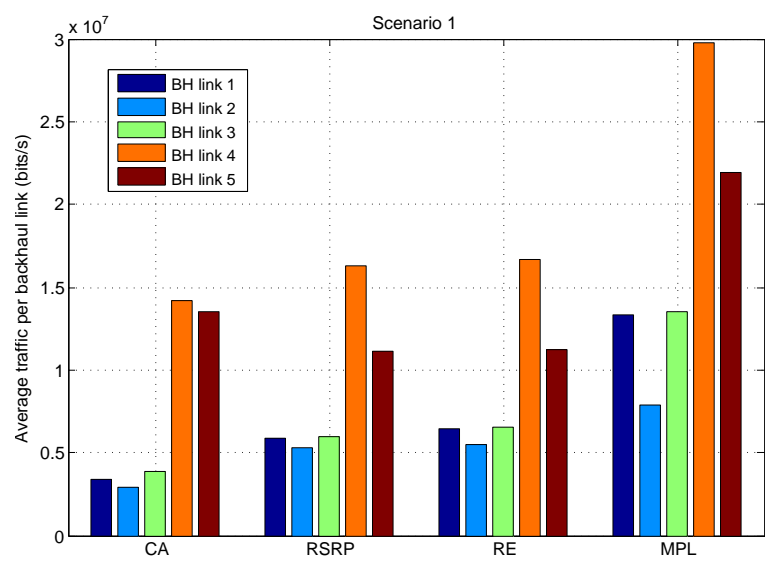

(b) Average traffic of each backhaul link for scenario 1 for $N=100$ UEs.

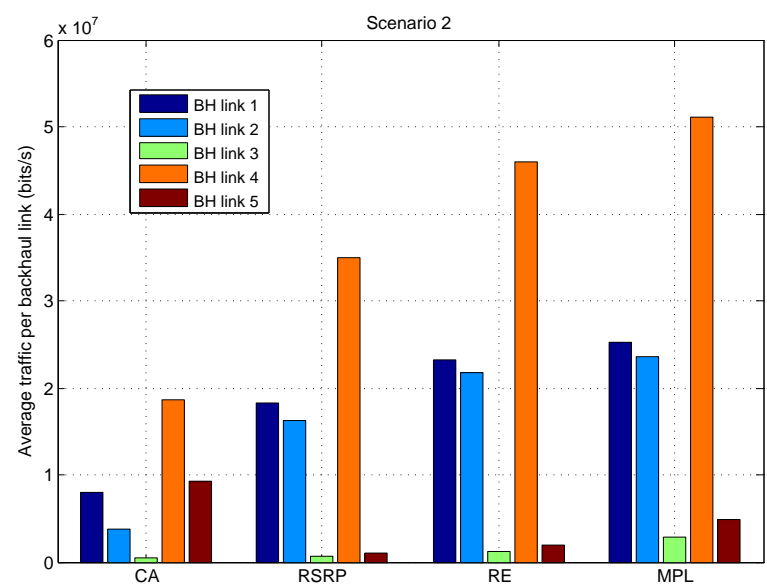

(d) Average traffic of each backhaul link for scenario 2 when $N=100$ UEs.

Fig. 4. Performance evaluation results for the simulation scenario with $\mathrm{BH}$ architecture 1, depicted in Fig. 3(a).

lower. This is better explained in Fig. 4(d), where the average traffic of each $\mathrm{BH}$ link is depicted for scenario 2, when $N=100$ UEs. As it is shown, CA achieves again much better BH load balancing (e.g., the UEs around SC 4 that have as a candidate cell the SC 5, will be connected to the SC that has the least loaded $\mathrm{BH}$ route). Then, as far as RE is concerned, it has poor energy efficiency performance, since there are more UEs associated to SCs than in RSRP and thus it presents higher BH energy consumption. Finally, MPL presents the lowest energy efficiency, since most UEs are associated with the closest SCs, resulting in the most highly loaded BH links (see Fig. 4(d)) and consequently in the highest BH energy consumption.

When the BH architecture scenario of Fig. 3(b) is applied, the total network energy efficiency, under the same user distribution scenarios (Fig. 5(a) and 5(c)), is lower than in the previous BH architecture scenario. This is due to the fact that the number of hops has a strong impact on the $\mathrm{BH}$ energy consumption (the SC 3 and SC 5 are now three hops away from the CN). Thus, apart from the need for a careful design of the $\mathrm{BH}$ architecture, backhaul-aware user association strategies should be developed, with the aim of achieving high network energy efficiency.

In Fig. 5(b) and 5(d), the average traffic of each BH link for $N=100$ UEs for the $\mathrm{BH}$ architecture of Fig. 3(b) is depicted, when scenario 1 and 2 are applied, respectively. In comparison to Fig. 4(b) and 4(d), it can be observed that the BH traffic distribution may differ significantly, when the $\mathrm{BH}$ architecture changes. This fact justifies the need for backhaul-aware user association strategies, especially in dense deployment scenarios. To that end, unlike the rest of the algorithms, CA takes into account both the access 


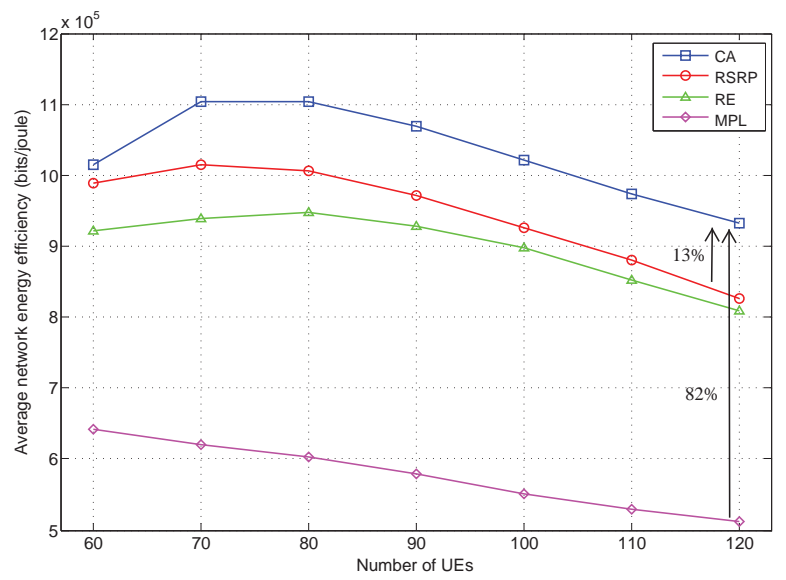

(a) Average network energy efficiency for different number of users $(N)$, when the UEs are uniformly distributed in the sector area (scenario 1).

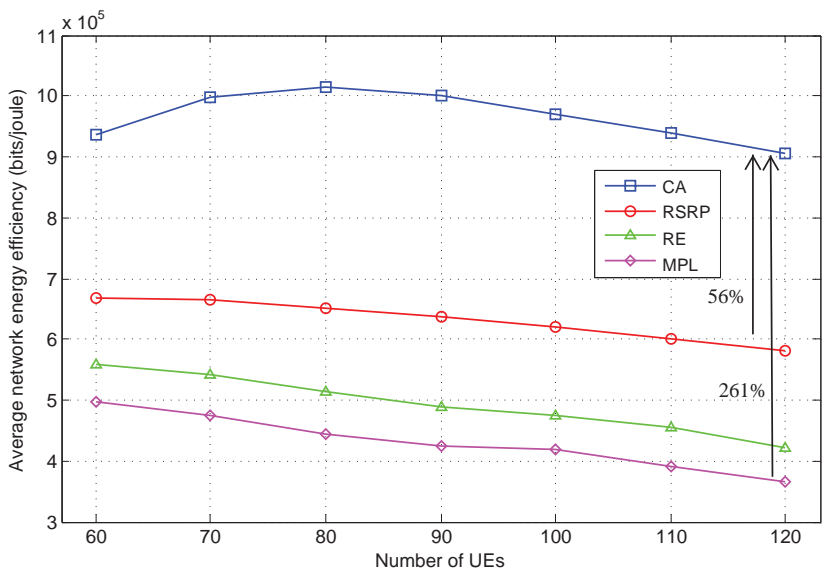

(c) Average network energy efficiency for different number of users $(N)$, for hotspot traffic (scenario 2).

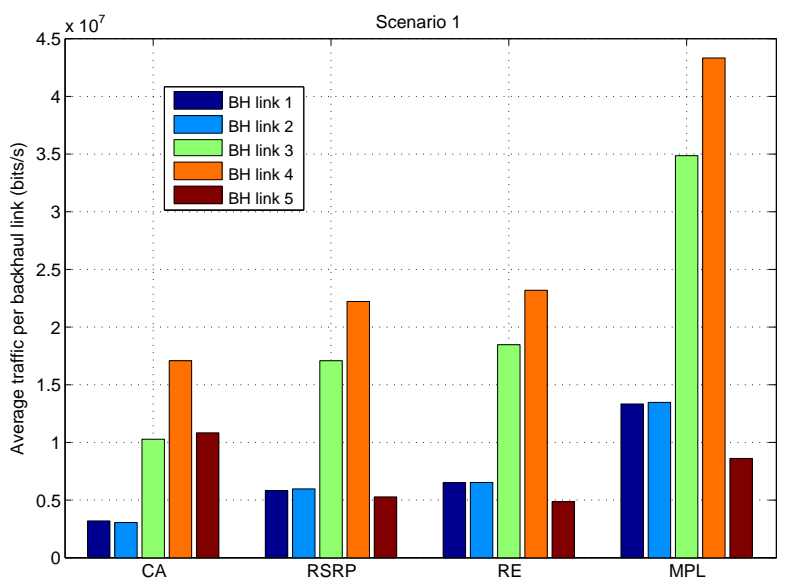

(b) Average traffic of each backhaul link for scenario 1 for $N=100$ UEs.

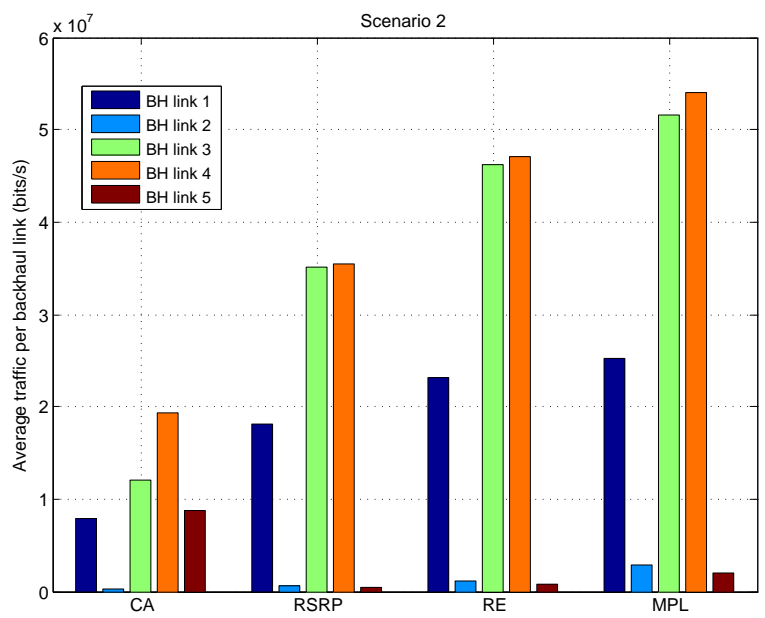

(d) Average traffic of each backhaul link for scenario 2 when $N=100$ UEs.

Fig. 5. Performance evaluation results for the simulation scenario with $\mathrm{BH}$ architecture 2, depicted in Fig. 3(b).

network and $\mathrm{BH}$ information in a dynamic way to achieve energy efficiency gains. For instance, notice that in the previous $\mathrm{BH}$ architecture, load balancing was applied between $\mathrm{BH}$ links 1, 2 and 3, whereas in this architecture, between BH links 1 and 2, since they are the same number of hops away from the $\mathrm{CN}$. It is also worth noting that due to its backhaul-awareness, CA could also deal with BH link failures.

In Fig. 6, the average network spectrum efficiency is depicted for all algorithms. This metric was shown to be independent of the applied BH architecture scenario. However, it highly depends on UEs' SINR and consequently on UEs' distribution. As it is shown in Fig. 6, CA achieves equally high spectrum efficiency to RSRP and RE for both user distribution scenarios, since it achieves the same throughput with the same amount of total RBPs used. In particular, the high spectrum efficiency of CA, stems from basing the user association not only on the BH configuration, but also on the link quality (i.e., CA considers as candidate cells for a UE only the cells with the highest SINR, that satisfy the UE requirements). MPL, unlike the rest of the algorithms, presents much lower spectrum efficiency, as it associates the UEs independently of their SINR. Thus, a UE may be associated with a SC, having the minimum path loss, although its received SINR is lower, and therefore more RBPs will be needed to achieve the same throughput. Moreover, notice that MPL achieves higher spectrum efficiency in scenario 2 than in scenario 1 . This is due to the fact that UEs that form a hotspot around a SC will have both low path loss and high SINR received from it, and thus will need a lower number of RBPs to satisfy their requirements. 


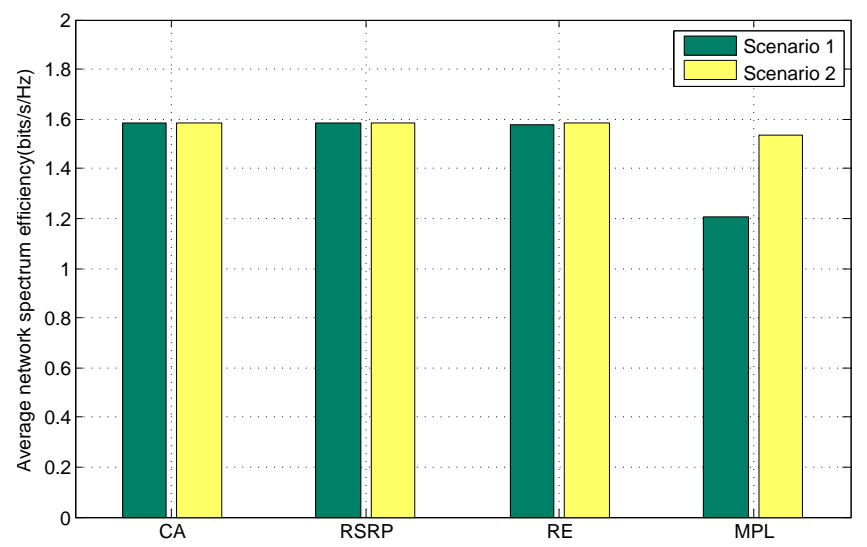

Fig. 6. Average network spectrum efficiency for both UE traffic distribution scenarios.

\section{CONCLUSION}

In this article, we studied the user association problem in future cognitive HetNets, where several $\mathrm{SCs}$ forward their traffic through the $\mathrm{BH}$ to the neighboring SCs until it reaches the $\mathrm{CN}$. Interference management, spectrum efficiency maximization and energy efficiency maximization were identified as the main technical challenges in this field that future user association solutions should meet. The performance of the existing user association algorithms was evaluated in terms of energy efficiency and spectrum efficiency under two different user distribution scenarios. The BH energy consumption was shown to play a key role in the overall network energy efficiency, thus predicating the development of energy-efficient backhaul-aware user association strategies. To that end, we proposed a context-aware algorithm, that takes into account both the access network and $\mathrm{BH}$ conditions to associate the UEs in an energy-efficient way, while achieving up to two and a half times more total energy efficiency than the reference algorithms.

\section{ACKNOWLEDGMENT}

This work has been funded by the Research Projects GREENET (PITN-GA-2010-264759), GREENT (CP8-006), GEOCOM (TEC2011-27723-C02-01), COST-TERRA (IC0905) and CROSSFIRE (MITN317126).

\section{REFERENCES}

[1] Small Cell Forum, "Backhaul Technologies for Small Cells: Use Cases, Requirements and Solutions".

[2] 3GPP TS 36.300 , “EUTRA \& EUTRAN Overall Description,” v. 11.5.0 Rel. 11.

[3] NGMN Alliance, "Small Cell Backhaul Requirements," v. 1.0, Jun. 2012.

[4] Tellabs, "Small cells address the growing demand for data," 2012.

[5] 3GPP TS 36.942, "Radio Frequency (RF) system scenarios,” v. 11.0.0, Rel. 11.

[6] D. Fooladivanda and C. Rosenberg, “Joint resource allocation and user association for heterogeneous wireless cellular networks," IEEE Trans. Wireless Commun., vol. 12, no. 1, pp. 248-257, Oct. 2012.

[7] H. S. Dhillon et al., "Modeling and Analysis of K-Tier Downlink Heterogeneous Cellular Networks," IEEE J. Sel. Areas Commun., vol. 30, no. 3, pp. 550-560, Apr. 2012.

[8] NTT DOCOMO, “Performance of eICIC with Control Channel Coverage Limitation,” R1-103264, 3GPP Std., May 2010.

[9] A. Damnjanovic et al., “A Survey on 3GPP Heterogeneous Networks,” IEEE Wireless Commun., vol. 18, no. 3, pp. 10-21, Jun. 2011.

[10] K.-C. Huang and Z. Wang, "Millimeter Wave Communication Systems," Wiley, 2011.

[11] 3GPP TS 36.814, “Further advancements for E-UTRA physical layer aspects,” v. 9.0.0, Mar. 2010.

Agapi Mesodiakaki obtained her five-year diploma (MSc) in Electrical and Computer Engineering in National Technical University of Athens (NTUA) in February 2011. During her studies, she accomplished a couple of internships in Hellenic Telecommunications Organization (OTE S.A) in Greece and in TU Darmstadt in Germany. Currently, she is a Marie-Curie Early-Stage-Researcher, pursuing a Ph.D. degree at the Signal Theory and Communications Department (TSC) of the Universitat Politenica de Catalunya (UPC) in Barcelona. 
Ferran Adelantado received the degree in Telecommunications Engineering from Universitat Politcnica de Catalunya (UPC) in December 2001. In June 2002 he joined the Radio Communications Research Group in UPC, where he completed his Ph.D. in May 2007. Since December 2007 he has been working as an Associate professor at Universitat Oberta de Catalunya (UOC). Also from Universitat Oberta de Catalunya (UOC), he received the BS. in Business Sciences in July 2012. He has participated in several funded European and National projects, such as SOFIA, COSMOS, EVEREST, CAUTION, AlgotithmsForRealRouting or CROSSFIRE.

Luis Alonso obtained the Ph.D. from UPC (Barcelona) in 2001 and got a permanent tenured position at the same University becoming an Associate Professor in 2006. He has been co-founder of the Wireless Communications and Technologies Research Group (WiComTec), to which currently belongs. He is author of forty research papers in international journals and magazines, one book, twelve chapters of books and more than one hundred papers in international congresses and symposiums. He has received several best paper awards.

Christos Verikoukis got his Ph.D. from the Technical University of Catalonia in 2000. He is currently a Senior Researcher and the Head of the SMARTECH department at CTTC and an adjunct professor at UB. He has published 60 journal papers and over 130 conference papers. He is also co-author in 2 books, 14 chapters in different books and in 2 patents. He has supervised 15 Ph.D. students and 5 Post Docs researchers since 2004. Dr. Verikoukis has participated in more than 30 competitive projects while he has served as the Principal investigator in national projects in Greece and Spain as well as the technical manager in Marie-Curie and Celtic projects. He has appointed to serve as a reviewer in FP7 projects funded by the European Commission and as an EU-independent expert acting evaluator in different FP7 funding mechanisms. He was General Chair of the 17th and 18th IEEE Workshop on Computer-Aided Modeling, Analysis and Design of Communication Links and Networks (CAMAD), and the TPC Co-Chair of the 15th IEEE International Conference on E-health Networking, Application \& Services (Healthcom). He is currently officer (Secretary) of the IEEE ComSoc Technical Committee on Communication Systems Integration and Modeling (CSIM). Dr. Verikoukis received the best paper award of the Communication QoS, Reliability \& Modeling Symposium (CQRM) symposium in the IEEE ICC11 conference and the EURASIP 2013 Best Paper Award for the Journal on Advances in Signal Processing. 